Internist

https://doi.org/10.1007/s00108-020-00905-x

Online publiziert: 19 . November 2020

(C) Der/die Autor(en) 2020, korrigierte

Publikation 2021

\section{Redaktion}

H. Haller, Hannover (Schriftleitung)

B. Salzberger, Regensburg

C.C. Sieber, Nürnberg

\section{Anamnese}

Die Erstvorstellung des 59-jährigen Patienten erfolgte über die zentrale Notaufnahme unserer Klinik in Begleitung seiner Ehefrau. Er hatte stärkste, seit einigen Tagen bestehende Oberbauchschmerzen. Am Vortag war der Patient von einer Urlaubsreise aus Österreich zurückgekehrt. Bei dort begonnener Symptomatik war eine Gastroskopie ohne wegweisenden Befund geblieben. Bei bereits länger bestehendem Halswirbelsäulen(HWS)-Syndrom wurde eine Therapie mit dem nichtsteroidalen Antirheumatikum Ibuprofen unter Säureblockade (Pantoprazol) eingeleitet.

Bei Aufnahme in domo wurden die Schmerzen als massiv und brennend beschrieben mit vermehrtem Auftreten in der Nacht sowie Ausstrahlung in den Rücken. Gewichtsverlust und Nachtschweiß wurden verneint.

An Vorerkrankungen bestanden neben dem HWS-Syndrom ein arterieller Hypertonus unter einer Kombinationstherapie mit Nebivolol und Olmesartan sowie eine zurückliegende depressive Episode. Operative abdominelle Eingriffe waren bislang nicht erfolgt.

\section{Klinischer Befund}

Bei Aufnahme zeigte sich der 59-jährige Patient in reduziertem Allgemeinzustand bei normalem Ernährungszustand $(82 \mathrm{~kg}$ bei $188 \mathrm{~cm}$, Body-Mass-In-

\author{
S. Welland ${ }^{\prime} \cdot$ C. Janssen ${ }^{2} \cdot$ K. I. Ringe ${ }^{3} \cdot$ G. Höglinger ${ }^{2} \cdot$ M. P. Manns ${ }^{1}$. \\ Ingmar Mederacke ${ }^{1}$ \\ 'Klinik für Gastroenterologie, Hepatologie und Endokrinologie, Medizinische Hochschule Hannover, \\ Hannover, Deutschland \\ ${ }^{2}$ Klinik für Neurologie, Medizinische Hochschule Hannover, Hannover, Deutschland \\ ${ }^{3}$ Institut für Diagnostische und Interventionelle Radiologie, Medizinische Hochschule Hannover, \\ Hannover, Deutschland
}

\title{
Massivste epigastrische Schmerzen bei einem 59-jährigen Patienten
}

$\operatorname{dex} 23,2 \mathrm{~kg} / \mathrm{m}^{2}$ ). Die Vitalparameter waren normwertig und es bestand kein Fieber. In der körperlichen Untersuchung zeigte sich ein druckschmerzhaftes Epigastrium ohne Zeichen eines Peritonismus. Stuhlgang und Miktion waren unauffällig.

\section{Diagnostik}

\section{Labordiagnostik}

Im Aufnahmelabor inklusive Urinuntersuchung ( $\delta$-Aminolävulinsäure und Porphobilinogen) zeigte sich abgesehen von einer milden normozytär-normochromen Anämie sowie einer isolierten, geringgradigen Erhöhung der Aspartat-Aminotransferase ein unauffälliger Befund. Serologisch wurden eine akute Infektion mit Herpesviren (Herpessimplex-Virus, Varizella-Zoster-Virus, Zytomegalievirus [CMV], Epstein-BarrVirus [EBV]) oder Hepatitisviren sowie eine Borrelieninfektion bei anamnestisch bestehendem Zeckenbiss ausgeschlossen.

\section{Sonographie}

Sonographisch ergaben sich bei bisher symptomloser Cholezystolithiasis lediglich eine leichte Prostatahypertrophie sowie minimale Mengen freier Flüssigkeit (Recessus hepatorenalis und splenorenalis).

\section{Endoskopie}

Eine erneute Gastroskopie erbrachte ein in Abheilung befindliches Ulkus im Bereich der Z-Linie sowie eine leichtgradige Refluxösophagitis (Los-Angeles-Stadium A) ohne weitere pathologische Befunde.

\section{Kardiale Diagnostik}

Bei unauffälligem Elektrokardiogramm konnte ein Myokardinfarkt laborchemisch ausgeschlossen werden. Echokardiographisch zeigte sich eine gute Pumpfunktion ohne Klappenvitien. Computertomographie(CT)-morphologisch ergaben sich keine Hinweise auf eine Lungenarterienembolie oder eine Aortendissektion.

\section{Magnetresonanztomographie}

Bei opiatrefraktären Schmerzen wurde Magnetresonanz(MR)-morphologisch ein die Perineuralscheide infiltrierendes Pankreaskarzinom ausgeschlossen (- Abb. 1). Bei zwischenzeitlich mitbestehenden Schmerzen in beiden proximalen Oberschenkeln ergab eine Magnetresonanztomographie der Wirbelsäule eine deutliche Kontrastmittelanreicherung der lumbalen Nervenwurzeln im Sinne einer Radikulitis (• Abb. 1). 

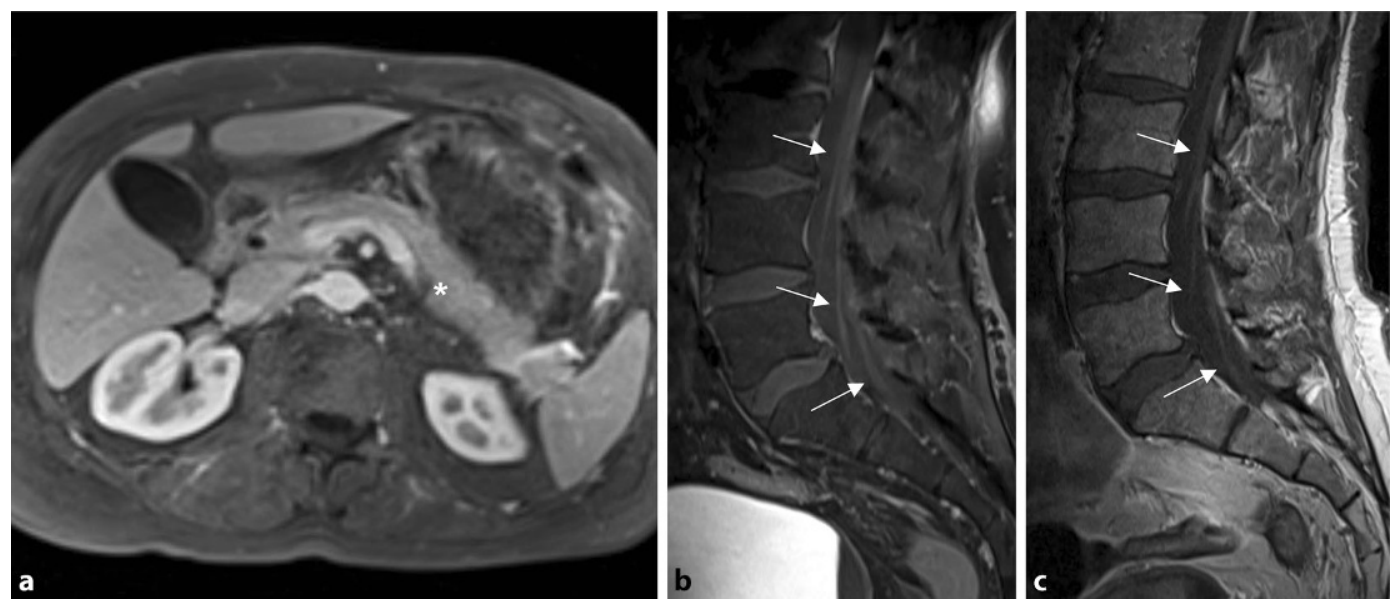

Abb. $1 \Delta$ Axiale kontrastmittelverstärkte Magnetresonanztomographie (MRT) in der portalvenösen Phase (a) mit unauffälligem Erscheinungsbild der Oberbauchorgane, insbesondere der Bauchspeicheldrüse (Sternchen). In der sagittalen T1-gewichteten MRT der Lendenwirbelsäule (b) ist eine pathologische Kontrastmittelaufnahme durch die Cauda equina erkennbar (Pfeile in b), die mit der Diagnose einer Radikulitis vereinbar ist. In der Kontrolle nach 3 Wochen (kontrastverstärkte sagittale T1-gewichtete MRTohne Fettsättigung; $c$ ) ist ein rückläufiges, aber noch geringes Enhancement erkennbar (Pfeile in c)
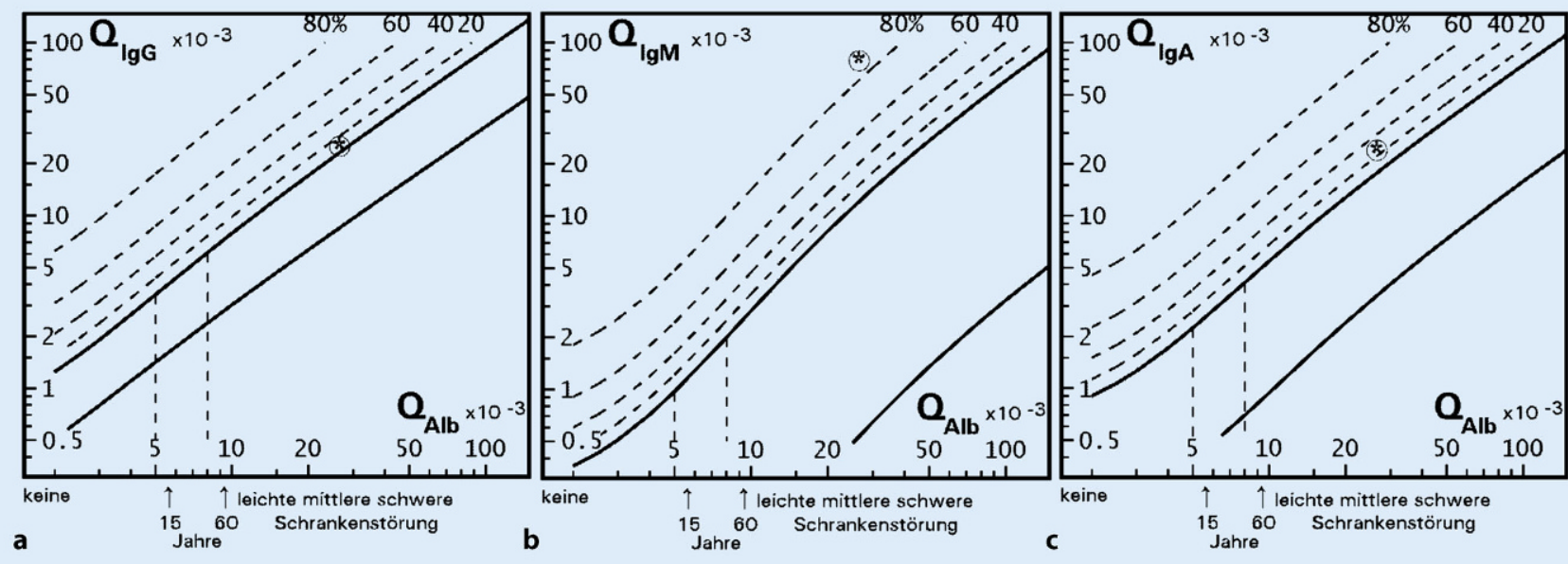

Abb. $2 \Delta$ Reiber-Schema bei Diagnosestellung für $\operatorname{lgG}(\mathbf{a}), \lg M(\mathbf{b})$ und $\lg A(\mathbf{c})$ im Liquor: deutlich erhöhter Albuminquotient als Ausdruck einer schweren Schrankenstörung im Rahmen einer entzündlichen Erkrankung des zentralen Nervensystems mit zusätzlich intrathekaler Antikörpersynthese von IgM und IgA. Alb Albumin, IgA Immunglobulin A, IgG Immunglobulin G, IgM Immunglobulin M, Q Quotient. Asterisk (*) entspricht dem gemessenen Wert für das entsprechende Immunglobulin des Patienten

\section{Liquordiagnostik}

Die Untersuchung des Liquors zeigte ein entzündliches Zellbild mit lymphomonozytärer Pleozytose, schwerer Schrankenstörung und intrathekaler Immunglobulinsynthese mit oligoklonalen Banden von Typ 2 (• Tab. 1; • Abb. 2), vereinbar mit einem infektiösen Geschehen passend zu einer Neuroborreliose. Die Diagnose konnte schließlich durch den Nachweis von borrelienspezifischen Antikörpern im Liquor bei persistierend negativer serologischer Untersuchung im Blut bestätigt werden ( $\bullet$ Tab. 2). Eine nachträglich durchgeführte Bestimmung von Borrelienantikörpern im Serum mittels Immunblot ergab zum Zeitpunkt der Diagnosestellung einen positiven Befund für eine singuläre Bande (Immunglobulin G gegen „variable major protein-like sequence, expressed“ [VlsE]).

\section{Diagnose}

\section{- Neuroborreliose nach Zeckenbiss mit diffuser Schmerzsymptomatik}

\section{Therapie und Verlauf}

Bei entzündlichem Liquorbefund wurde eine empirische antiinfektive Therapie mit Ceftriaxon und Aciclovir begonnen. Unter Erwägung einer autoimmunologischen Ursache der nachgewiesenen axonalen Polyneuropathie wurde ein Stero- 
idstoß mit Methylprednisolon ergänzt. Mit Erhalt des Nachweises von Borrelienantikörpern im Liquor wurde die antiinfektive Therapie mit Ceftriaxon über insgesamt 14 Tage intravenös durchgeführt. Unter einer Medikation mit Pregabalin war der Patient erstmalig symptomfrei. Eine Woche nach Beendigung der antiinfektiven Therapie erfolgte eine erneute stationäre Aufnahme bei neu aufgetretener Hypästhesie und Kribbelparästhesien im linken Bein (Dermatome L5-S1 links). Bei rückläufiger, aber weiterhin erhöhter Liquorzellzahl (• Tab. 1) sowie MR-morphologischer Kontrastmittelanreicherung in der Cauda equina konnte nach erneuter 14-tägiger Therapie mit Ceftriaxon eine anhaltende Remission erreicht werden.

\section{Diskussion}

\section{Differenzialdiagnosen bei abdominalen Schmerzen}

Oftmals führen bei epigastrischen Schmerzen eine gründliche Anamnese und die klinische Untersuchung schon zu einer Verdachtsdiagnose, die durch gut verfügbare, wenig invasive und kosteneffektive apparative Maßnahmen bestätigt oder ausgeschlossen werden kann. So zählt zur Abklärung neben der laborchemischen Basisdiagnostik eine Sonographie des Abdomens, die Aufschluss über strukturelle Veränderungen an den Bauchorganen (z.B. Raumforderungen) oder Hinweise auf Gefäßverschlüsse geben kann. Die Aussagekraft einer Sonographie kann jedoch durch Faktoren wie das Körpergewicht, Immobilität des Patienten, Meteorismus oder fehlende Routine des Untersuchers limitiert sein. Zum Ausschluss eines Aortenaneurysmas, einer Dissektion oder einer mesenterialen Ischämie ist daher oftmals eine CT notwendig. Im weiteren Vorgehen gehört auch eine Gastroskopie zur Abklärung epigastrischer Beschwerden.

Nach Ausschluss häufiger Ursachen abdomineller Schmerzen müssen auch seltenere Ursachen, wie eine Porphyrie, in Betracht gezogen werden. Auch hämatologische Grunderkrankungen (Leukämien, hämolytische Anämien wie Sichelzellanämie oder Sphärozytose) soll- ten durch Anfertigung eines Differenzialblutbilds mit Blutausstrich zur mikroskopischen Beurteilung ausgeschlossen werden. Selten kann ein Pankreaskarzinom im frühen Stadium durch eine Perineuralscheideninfiltration zu ausgeprägten Oberbauchbeschwerden führen; eine solche Infiltration ist am besten MRmorphologisch zu erfassen.

Auch metabolische Störungen wie die Hyperkalzämie, eine Pseudoperitonitis diabetica im Rahmen eines Diabetes mellitus, eine thyreotoxische Krise oder Addison-Krise sowie eine Urämie sollten ausgeschlossen werden, wobei die Laboruntersuchung hier wegweisend ist. Hinweise auf eine Bleivergiftung als sehr seltene Ursache, die neben Bauchschmerzen auch zu auffälliger Färbung der Gingiva, Einschränkungen der Hämatopoese und Radialislähmungen führen kann, geben Erythrozyten mit basophiler Tüpfelung.

\section{》) Auch Neuropathien können ursächlich für anders nicht zu erklärende abdominelle Schmerzen sein}

Zuletzt können auch Neuropathien ursächlich für anders nicht zu erklärende abdominelle Schmerzen sein. Im Falle von dermatombezogenen Schmerzen muss auch bei fehlender typischer Effloreszenz ein Herpes zoster serologisch ausgeschlossen werden [1]. Auch infektiologische Ursachen wie eine Infektion mit "human immunodeficiency virus", CMV oder EBV sowie Hepatitis B, C oder E, aber auch bakterielle Erreger wie Mykoplasmen, Borrelien oder das Clostridioides-difficile-Toxin können eine akut verlaufende Neuropathie hervorrufen [2, 3]. Auch die Neuroborreliose, ausgelöst durch eine Polyradikulitis spinaler Nerven, stellt eine mögliche seltene Ursache für abdominelle Schmerzen dar.

\section{Epidemiologie der Neuroborreliose}

Häufig kann eine genaue Anamnese bei einem Zeckenbiss Hinweise auf die Wahrscheinlichkeit einer Borrelieninfektion liefern. Bereits Daten aus den
Internist

https://doi.org/10.1007/s00108-020-00905-x

(c) Der/die Autor(en) 2020

\section{Massivste epigastrische Schmerzen bei einem 59- jährigen Patienten}

\section{Zusammenfassung}

Abdominelle Schmerzen sind oft Konsultationsanlass in Arztpraxen und Notaufnahmen. Die häufigsten Differenzialdiagnosen lassen sich mit gut verfügbarer, kosteneffektiver und risikoarmer Diagnostik (Laboruntersuchungen, Sonographie, Gastroskopie) bestätigen. Zum Ausschluss seltener Ursachen, wie kleiner solider oder hämatologischer Malignome, Stoffwechselstörungen oder Polyneuropathien unterschiedlichster Genese, kann eine erweiterte Diagnostik erforderlich sein. Im Folgenden stellen wir den Fall eines Patienten mit massivsten epigastrischen Beschwerden infolge einer Neuroborreliose vor und rekapitulieren die diagnostischen Schritte zur Abklärung des abdominellen Schmerzes.

\section{Schlüsselwörter}

Akute Neuroborreliose - Radikulitis . Polyneuropathien - Erythema chronicum migrans · Liquordiagnostik

\section{Severe epigastric pain in a 59- year-old patient}

\section{Abstract}

Abdominal pain is a frequent cause of consultation to doctors' offices and emergency rooms. The most common differential diagnoses can be confirmed with readily available, cost-effective, and low-risk diagnostic tools such as laboratory tests, ultrasound, or gastroscopy. Additional diagnostic tests are required to exclude rare causes such as small, solid, or hematological malignancies, metabolic disorders, or polyneuropathies of varying origin. In the following, we present the case of a patient with severe epigastric pain due to neuroborreliosis, and recapitulate the diagnostic steps for clarifying abdominal pain using this example.

\section{Keywords}

Lyme neuroborreliosis, acute - Radiculitis . Polyneuropathies - Erythema chronicum migrans · Diagnostics, cerebrospinal fluid 


\section{Kasuistiken}

Tab. 1 Liquoranalyse bei Diagnosestellung sowie 3 Wochen nach Therapiestart

\begin{tabular}{|c|c|c|c|c|}
\hline & $\begin{array}{l}\text { Zeitpunkt } \\
\text { Diagnose }\end{array}$ & $\begin{array}{l}3 \text { Wochen nach } \\
\text { Therapiestart }\end{array}$ & Einheit & $\begin{array}{l}\text { Referenz- } \\
\text { wert }\end{array}$ \\
\hline \multicolumn{5}{|l|}{ Zytologie } \\
\hline Zellzahl Liquor & 70 & 31,3 & Zellzahl/ $\mu$ l & $<5,0$ \\
\hline Erythrozyten & 1,0 & 0,3 & Zellzahl/ $\mu$ l & $<1,0$ \\
\hline Lymphozyten & 89 & 98 & $\%$ & - \\
\hline Monozyten & 10 & 5 & $\%$ & - \\
\hline Granulozyten & 0 & 0 & $\%$ & - \\
\hline Plasmazellen & 1 & 0 & $\%$ & - \\
\hline \multicolumn{5}{|l|}{ Proteine } \\
\hline Gesamtprotein & 1713 & 598 & $\mathrm{mg} / \mathrm{l}$ & - \\
\hline Liquor-Alb & 1060 & 460 & $\mathrm{mg} / \mathrm{l}$ & - \\
\hline Liquor-lgG & 206 & 54,6 & $\mathrm{mg} / \mathrm{l}$ & - \\
\hline Liquor-lgA & 51,9 & 12,4 & $\mathrm{mg} / \mathrm{l}$ & - \\
\hline Liquor-IgM & 51,1 & 6,8 & $\mathrm{mg} / \mathrm{l}$ & - \\
\hline Serum-Alb & 39,9 & 42,3 & $g / l$ & - \\
\hline Serum-lgG & 8,21 & 8,8 & $g / l$ & - \\
\hline Serum-IgA & 2,18 & 2,23 & $g / l$ & - \\
\hline Serum-IgM & 0,65 & 0,86 & $g / l$ & - \\
\hline Q-Alb & 26,57 & 10,87 & - & - \\
\hline Q-lgG & 25,09 & 6,19 & - & - \\
\hline Q-IgA & 23,81 & 5,56 & - & - \\
\hline Q-IgM & 78,50 & 7,99 & - & - \\
\hline IgG-Index & 0,944 & 0,569 & - & - \\
\hline Lokale Synthese IgA & 25,7 & 0,0 & $\%$ & - \\
\hline Lokale Synthese IgG & 7,9 & 0,0 & $\%$ & - \\
\hline Lokale Synthese IgM & 84,5 & 59,5 & $\%$ & - \\
\hline Oligoklonale Banden & Typ 2 & Typ 3 & - & - \\
\hline
\end{tabular}

Diagnose Therapiestart

Einheit

Tab. 2 Borreliendiagnostik im Serum und Liquor

\begin{tabular}{|c|c|c|c|c|}
\hline & $\begin{array}{l}\text { Zeitpunkt } \\
\text { Diagnose }\end{array}$ & Einheit & Bewertung & $\begin{array}{l}\text { Interpretations- } \\
\text { bereich }\end{array}$ \\
\hline \multicolumn{5}{|l|}{ Serum } \\
\hline $\begin{array}{l}\text { Borrelia-burgdorferi-Komplex-lgG } \\
\text { (ELISA) }\end{array}$ & 13,49 & $\mathrm{U} / \mathrm{ml}$ & Negativ & $\begin{array}{l}<20 \text { (grenzwertig: } \\
20-24 \text { ) }\end{array}$ \\
\hline $\begin{array}{l}\text { Borrelia-burgdorferi-Komplex-lgM } \\
\text { (ELISA) }\end{array}$ & 16,97 & $\mathrm{U} / \mathrm{ml}$ & Negativ & $\begin{array}{l}<20 \text { (grenzwertig: } \\
20-24 \text { ) }\end{array}$ \\
\hline \multicolumn{5}{|l|}{ Liquor } \\
\hline $\begin{array}{l}\text { Borrelia-burgdorferi-Komplex-lgG } \\
\text { (ELISA) }\end{array}$ & - & - & Positiv & Negativ \\
\hline $\begin{array}{l}\text { Borrelia-burgdorferi-Komplex-lgM } \\
\text { (ELISA) }\end{array}$ & - & - & Positiv & Negativ \\
\hline $\begin{array}{l}\text { Borrelia-Liquor/ } \\
\text { Serum-Antikörperindex für lgG }\end{array}$ & 15,18 & - & Positiv & $\begin{array}{l}\leq 1,3 \text { (grenzwertig: } \\
1,3-1,5)\end{array}$ \\
\hline $\begin{array}{l}\text { Borrelia-Liquor/ } \\
\text { Serum-Antikörperindex für lgM }\end{array}$ & 15,92 & - & Positiv & $\begin{array}{l}\leq 1,3 \text { (grenzwertig: } \\
1,3-1,5)\end{array}$ \\
\hline
\end{tabular}

1990er-Jahren belegen eine deutlich höhere Inzidenz der Lyme-Borreliose in Österreich mit etwa 120 Erkrankten pro 100.000 Einwohner im Vergleich zu Deutschland mit 26-41 Fällen pro 100.000 Einwohner [4]. Da der Holzbock als übertragende Zecke ab einer Außentemperatur von $6^{\circ} \mathrm{C}$ aktiv ist, erfolgt eine Übertragung in den Monaten von Februar/März bis Oktober/November mit einem Häufigkeitsgipfel der Neuroborreliose in den Monaten Juli und August [4, 5].

\section{Klinische Manifestation}

Eine Neuroborreliose tritt bei etwa $10-15 \%$ aller klinisch apparenten Borrelieninfektionen auf. Die häufigste Manifestationsform mit etwa $10 \%$ stellt eine Beteiligung des peripheren Nervensystems mit inflammatorischer Polyradikulitis von Spinalnerven oder Hirnnerven mit segmental-radikulärer, häufig nächtlicher Schmerzsymptomatik in Form des Bannwarth-Syndroms dar, in 2-4\% der Fälle kann es zudem zu einer Beteiligung des zentralen Nervensystems im Sinne einer Meningitis oder seltener einer Myelitis oder Enzephalitis kommen. Begleitend können weitere Symptome wie Kopfschmerz, Fatigue, Parästhesien sowie periphere Nervenlähmungen der Extremitäten oder Hirnnerven (häufig des N. facialis) bestehen [6]. Typischerweise sprechen die Schmerzen schlecht bis gar nicht auf eine herkömmliche analgetische Therapie an und werden wie neuropathische Schmerzen als brennend und bohrend beschrieben [7]. Symptome treten in einem Zeitraum von 2 bis 18 Wochen nach Infektion auf (im Durchschnitt nach 4-6 Wochen; [8]). Nur in einem Drittel der Fälle ist ein Zeckenbiss sowie in $25-50 \%$ ein Erythema chronicum migrans erinnerlich. Bei der Mehrheit, jedoch nicht bei allen Patienten entwickeln sich im Verlauf von ein bis 4 Wochen überwiegend motorisch-neurologische Ausfälle, die hauptsächlich im Bereich der Hirnnerven lokalisiert sind [9]. 


\section{Diagnostik}

Im Serum können borrelienspezifische Antikörper gemessen werden, die mit einer Latenz von 4 bis 6 Wochen nach Infektion im peripheren Blut nachweisbar sind. Bei Patienten mit Erythema migrans kann in bis zu $50 \%$ der Fälle die serologische Antikörpertestung unauffällig bleiben [10]. Bei begründetem Verdacht auf eine Neuroborreliose sollte daher eine Liquorpunktion durchgeführt werden, in der sich typischerweise eine lymphozytäre Pleozytose mit Erhöhung des Gesamteiweißes im Sinne einer Schrankenstörung ergibt [11]. Die definitive Diagnose der Erkrankung erfolgt durch Nachweis einer intrathekalen Synthese borrelienspezifischer Antikörper.

\section{Therapie der akuten Neuroborreliose}

Da auch Jahre nach einer Borrelieninfektion noch Immunglobulin-M-Antikörper nachweisbar sein können, sollte die Indikation zur Therapie immer im klinischen Kontext gestellt werden. Insgesamt kann die Neuroborreliose durch die Gabe von Doxycyclin, Ceftriaxon, Cefuroxim oder Penicillin G gut therapiert werden. Die orale Doxycyclintherapie ist der parenteralen Therapie mit $\beta$-Laktam-Antibiotika nicht unterlegen.

Leitliniengerecht ist eine antiinfektive Therapie über eine Dauer von 14 Tagen bei früher Neuroborreliose empfohlen, da Studien bei Verlängerung der Therapiedauer keinen Vorteil zeigen konnten [12]. Ein Jahr nach Behandlung sind $90 \%$ der Patienten symptomfrei [13]. Bei chronischer Neuroborreliose sollte eine längere Therapie für 14-21 Tage angestrebt werden [14].

\section{Fazit für die Praxis}

Bei der Abklärung epigastrischer Beschwerden sollten nach Ausschluss häufiger Ursachen auch seltenere Differenzialdiagnosen wie die Neuroborreliose berücksichtigt werden, die insbesondere auch aufgrund der guten Therapierbarkeit nicht übersehen werden sollte. Eine Neuroborreliose kann auch ohne Erythema chronicum migrans auftreten, auch fällt die serologische Untersuchung auf Borrelien im Blut nicht immer positiv aus, sodass bei Verdacht eine Liquorpunktion zur Diagnosestellung erfolgen sollte.

\section{Korrespondenzadresse}

PD Dr. med. Ingmar Mederacke

Klinik für Gastroenterologie, Hepatologie und Endokrinologie, Medizinische Hochschule Hannover

Carl-Neuberg-Str. 1, 30625 Hannover,

Deutschland

mederacke.ingmar@mh-hannover.de

Funding. Open Access funding enabled and organized by Projekt DEAL.

\section{Einhaltung ethischer Richtlinien}

Interessenkonflikt. S. Welland, C. Janssen, K.I. Ringe, G. Höglinger, M.P. Manns und I. Mederacke geben an, dass kein Interessenkonflikt besteht.

Für diesen Beitrag wurden von den Autoren keine Studien an Menschen oder Tieren durchgeführt. Für die aufgeführten Studien gelten die jeweils dort angegebenen ethischen Richtlinien. Für Bildmaterial oder anderweitige Angaben innerhalb des Manuskripts, über die Patienten zu identifizieren sind, liegt von ihnen und/oder ihren gesetzlichen Vertretern eine schriftliche Einwilligung vor.

Open Access. Dieser Artikel wird unter der Creative Commons Namensnennung 4.0 International Lizenz veröffentlicht, welche die Nutzung, Vervielfältigung, Bearbeitung, Verbreitung und Wiedergabe in jeglichem Medium und Format erlaubt, sofern Sie den/die ursprünglichen Autor(en) und die Quelle ordnungsgemäßnennen, einen Link zur Creative Commons Lizenz beifügen und angeben, ob Änderungen vorgenommen wurden.

Die in diesem Artikel enthaltenen Bilder und sonstiges Drittmaterial unterliegen ebenfalls der genannten Creative Commons Lizenz, sofern sich aus der Abbildungslegende nichts anderes ergibt. Sofern das betreffende Material nicht unter der genannten Creative Commons Lizenz steht und die betreffende Handlung nicht nach gesetzlichen Vorschriften erlaubt ist, ist für die oben aufgeführten Weiterverwendungen des Materials die Einwilligung des jeweiligen Rechteinhabers einzuholen.

Weitere Details zur Lizenz entnehmen Sie bitte der Lizenzinformation auf http://creativecommons.org/ licenses/by/4.0/deed.de.

\section{Literatur}

1. Gross GE, Eisert L, Doerr HW et al (2020) S2k guidelines for the diagnosis and treatment of herpes zoster and postherpetic neuralgia. J Dtsch Dermatol Ges 18(1):55-78. https://doi.org/10. 1111/ddg.14013
2. Heuß D (2019) Leitlinie diagnostik bei polyneuropathien. https://www.dgn.org/leitlinien/3754II-030-067-diagnostik-bei-polyneuropathien2019.Zugegriffen:5. Aug. 2020

3. Dalton HR, Kamar N, van Eijk JJ etal (2016) Hepatitis E virus and neurological injury. Nat Rev Neurol 12(2):77-85. https://doi.org/10.1038/nrneurol. 2015.234

4. RKI (2019) Ratgeber Lyme-Borreliose. https:// www.rki.de/DE/Content/Infekt/EpidBull/ Merkblaetter/Ratgeber_LymeBorreliose.html. Zugegriffen: 5. Aug. 2020

5. Vogelgesang JR, Walter M, Kahl O, Rubel F, Brugger K (2020) Long-term monitoring of the seasonal density of questing ixodid ticks in vienna (austria): Setup and first results. Exp Appl Acarol 81(3):409-420. https://doi.org/10.1007/s10493020-00511-4

6. Ogrinc K, Lusa L, Lotrič-Furlan S et al (2016) Course and outcome of early european lyme neuroborreliosis (bannwarth syndrome): clinical and laboratory findings. clin Infect Dis 63(3):346-353. https://doi.org/10.1093/cid/ciw299

7. Reik L, Steere AC, Bartenhagen NH, Shope RE, Malawista SE (1979) Neurologic abnormalities of lyme disease. Medicine 58(4):281-294. https://doi. org/10.1097/00005792-197907000-00001

8. Halperin JJ (2013) Nervous system lyme disease: diagnosis and treatment. Curr Treat Options Neurol 15(4):454-464. https://doi.org/10.1007/s11940013-0240-y

9. Kaiser R (1994) Variable CSF findings in early and late lyme neuroborreliosis: a follow-up study in 47 patients. jNeurol 242(1):26-36. https://doi.org/ 10.1007/BF00920571

10. Halperin JJ (2017) Neuroborreliosis. j Neurol 264(6):1292-1297. https://doi.org/10.1007/ s00415-016-8346-2

11. Djukic M, Schmidt-Samoa C, Lange P et al (2012) Cerebrospinal fluid findings in adults with acute lyme neuroborreliosis. j Neurol 259(4):630-636. https://doi.org/10.1007/s00415-011-6221-8

12. Oksi J, Nikoskelainen J, Hiekkanen $\mathrm{H}$ et al (2007) Duration of antibiotic treatment in disseminated lyme borreliosis: a double-blind, randomized, placebo-controlled, multicenter clinical study. Eur J Clin Microbiol Infect Dis 26(8):571-581. https:// doi.org/10.1007/s10096-007-0340-2

13. Kaiser R (2004) Clinical courses of acute and chronic neuroborreliosis following treatment with ceftriaxone. Nervenarzt 75(6):553-557. https:// doi.org/10.1007/s00115-003-1560-z

14. Rauer $S$, Kastenbauer S, Fingerle V, Hunfeld KP, Huppertz HI, Dersch R (2018) Lyme neuroborreliosis. dtsch Arztebl Int 115(45):751-756 